\title{
Negative Feedback Neuroendocrine Control of the Inflammatory Response in Rats
}

\author{
Paul G. Green, ${ }^{7}$ Frederick J.-P. Miao, ${ }^{2}$ Wilfrid Jänig, ${ }^{5}$ and Jon D. Levine ${ }^{1,2,3,4}$ \\ Departments of ${ }^{1}$ Oral Surgery, ${ }^{2}$ Medicine, and ${ }^{3}$ Anatomy and ${ }^{4}$ Division of Neurobiology, University of California, San \\ Francisco, California 94143 and ${ }^{5}$ Physiologisches Institut, Christian-Albrechts-Universität, 24098 Kiel, Germany
}

We describe that an ongoing inflammatory response at one site (produced by complete Freund's adjuvant injection in the hindpaw) produces a negative feedback inhibition on plasma extravasation, produced by perfusion of the inflammatory mediator, bradykinin (160 nM), at a second site (the knee joint). This negative feedback process is abolished in rats that have been neonatally treated with capsaicin to deplete most of their unmyelinated primary afferent fibers, which suggests that this negative feedback process is mediated by activation of primary afferent fibers. Electrical stimulation of the hindpaw at intensities that excite C-fibers also inhibited bradykinin-induced plasma extravasation. Stimulation at intensities that only excite A-fibers had no effect on bradykinin-induced plasma extravasation. Platelet activating factor-induced plasma extravasation, which is not dependent on the innervation of the joint, was not inhibited by stimulation of C-fibers from the hindpaw.

Acute surgical interruption of the lumbar sympathetic outflow to the hind limb (the paravertebral ganglia between $L_{2}$ and $L_{4}$ ) did not attenuate the depression of bradykinininduced plasma extravasation produced by C-fiber stimulation. This indicates that the depression is not mediated by activity in the sympathetic outflow. Transection of the spinal cord, hypophysectomy, inhibition of corticosterone synthesis, and adrenalectomy (but not adrenal medullectomy) all prevented the inhibition of BK-induced plasma extravasation by electrical simulation, indicating that the negative feedback inhibition on plasma extravasation is dependent on an intact neuraxis and an intact hypothalamicpituitary-adrenocortical axis.

In summary, our data demonstrate a negative feedback inhibition of an inflammatory process, which is elicited by stimulation of $\mathbf{C}$-fiber afferents. It is mediated by ascending pathways in the spinal cord, and probably the hypothalamic-pituitary-adrenocortical axis.

[Key words: primary afferent nociceptor, sympathetic nervous system, hypothalamic-pituitary-adrenal axis, adrenal medulla, adrenal cortex, neurogenic inflammation, bradykinin, hypophysectomy, capsaicin, sympathectomy, spinal transection, adrenalectomy]

Received Noy. 9, 1994; revised Jan. 23, 1995; accepted Jan. 24, 1995

This work was supported by NIH Grant AR32634 and by the Arthritis Foundation (Northern California Chapter).

Correspondence should be addressed to Jon D. Levine, M.D., Ph.D., Department of Medicine, S-1334/Box 0152^, UCSF, San Francisco, C $\Lambda$ 94143 $0452 \mathrm{~A}$.

Copyright $@ 1995$ Society for Neuroscience 0270-6474/95/154678-09\$05.00/0
The inflammatory response is a physiological process that protects the organism against infection and repairs tissue after traumatic injury. The mechanisms which initiate the inflammatory response are well documented, and involve both humoral (Williams et al., 1988) and cellular (Keshav et al., 1990; Roos and Dolman, 1990; Haskill et al., 1992) components of the immune system, neuroendocrine mechanisms (Garcia-Leme et al., 1992; Sternberg et al., 1992), as well as sensory (Kowalski and Kaliner, 1988; Mantyh et al., 1989; Barnes, 1991) and autonomic components (Levine et al., 1987; Khalil and Helme, 1989; Kidd et al., 1992) of the peripheral nervous system (PNS). While inflammation is normally a tissue protective and/or reparative process, and beneficial to the organism (Basbaum and Levine, 1991), the inflammatory process itself, especially if prolonged, can lead to tissue injury, as in the presence of chronic inflammatory disease, such as rheumatoid arthritis. Thus, one would expect that there exists a mechanism(s) for the appropriate termination of the inflammatory response, and that deficiencies in such mechanisms might contribute to production of inflammatory disease (Calogero et al., 1992; Zelazowski et al., 1992).

While removal or cessation of the stimulus that has initiated an inflammatory response may be sufficient to terminate inflammation, there is also evidence that there are physiological mechanisms which produce negative feedback control of the inflammatory response. For example, the inflammatory response results, in part, from the opening of interendothelial gaps, which quickly close again, even though the permeability-inducing agents continue to be present (Casley-Smith and Window, 1976). Another mechanism that may act to inhibit or terminate the inflammatory response is activation of the hypothalamic-pituitary-adrenocortical (HPA) axis. Of interest, inflammation is known to cause local release of a number of substances, such as cytokines, that can act in the central nervous system to activate the HPA axis (Payne and Krueger, 1992; Dunn, 1993). Activation of the HPA axis, in turn leads to the release of substances, such as corticosterone, which act to suppress the inflammatory response (Sternberg et al., 1989; Sweep et al., 1991; Calogero et al., 1992). Additional evidence for a contribution of the HPA axis to the negative feedback control of inflammation comes from studies involving pharmacological, anatomical, and genctic lesions of the HPA axis. For example, there is an increased susceptibility to arthritis in Lewis rats that have a genetically determined corticotropin-releasing factor (CRF) deficiency and hyporesponsiveness to administered CRF (Sternberg et al., 1989), in adrenalectomized (Harbuz et al., 1993) or hypophysectomized (Neidhart and Fluckiger, 1992) rats, and in chickens with impaired HPA-axis function (Brezinschek et al., 
1990). A decreased susceptibility to inflammatory disease is, in fact, seen in CRF-hyperresponsive Fischer rats (Sternberg et al., 1990; Calogero et al., 1992; Zelazowski et al., 1992). In humans with rheumatoid arthritis, there is an altered HPA axis circadian rhythm and an abnormality of the HPA axis response to inflammatory stimuli (Neeck et al., 1990; Chikanza et al., 1992); however, it is unclear whether this is the consequence of rheumatoid arthritis or a causative factor in the generation of rheumatoid arthritis.

In this report, we describe the results of experiments which show that ongoing inflammation at one site, the hindpaw, produces a negative feedback inhibition of bradykinin (BK)-induced plasma extravasation (a component of inflammation) at another site, the knee joint. Our data demonstrate that this negative feedback inhibition is mediated by afferent $\mathrm{C}$-fibers and is dependent on spinal ascending pathways as well as an intact HPA axis.

\section{Materials and Methods}

\section{Animals}

The experiments were performed on 300-400 gm male Sprague-Dawley rats (purchased from Bantin and Kingman, Fremont $\mathrm{C} \Lambda$, except for hypophysectomized rats and their normal controls, which were purchased from Charles River, Hollister, CA). The rats were housed in a temperature and humidity controlled environment, and were maintained on a $12 \mathrm{hr}$ light/dark cycle (lights on at 06:00). Food and water were available ad libitum. Care and handling of animals was performed in accordance with 'The American Physiological Society guidelines.

\section{Plasma extravasation}

Rats were anesthetized with sodium pentobarbital (Anthony Products Co., Arcadia, CA; $65 \mathrm{mg} / \mathrm{kg}$ ). Skin overlying the knee was excised to expose the joint capsule, and rats were then given an intravenous injection of Evans blue dye $(50 \mathrm{mg} / \mathrm{kg}$, in a volume of $2.5 \mathrm{ml} / \mathrm{kg})$. A 30 gauge hypodermic needle was then inserted for the inflow of perfusion fluid $(250 \mu \mathrm{l} / \mathrm{min}$; controlled by a Syringe pump, Sage Instruments Model 341B), and after perfusion of 100-200 $\mu$ l of fluid, a second needle (25 gauge) was inserted into the joint cavity for outflow of the perfusion fluid ( $250 \mu \mathrm{l} / \mathrm{min}$; Syringe pump, Sage Instruments Model 351). Samples of perfusion fluid were collected every $5 \mathrm{~min}$ for a period of $70 \mathrm{~min}$, and samples were analyzed for Fivans blue dye concentration by spectrophotometric measurement of absorbance at $620 \mathrm{~nm}$, which is linearly related to dye concentration (Carr and Wilhelm, 1964). Following establishment of baseline levels of plasma extravasation in the first three samples, BK (160 nM) was added to the perfusing fluid and remained present in the fluid for the duration of the experiment.

\section{Freund's adjuvant-induced monoarthritis}

Inflammation at the primary site was induced by injecting rats with 150 $\mu l$ of complete Freund's adjuvant subcutaneously into the plantar surface of the hindpaw. This produces an inflammatory response beginning $46 \mathrm{hr}$ postinjection and lasting for several days. Fifteen hours after injection of Freund's adjuvant, BK-induced plasma extravasation was evaluated in the rat's knee joint.

\section{Electrical stimulation of the hindpaw}

Two stainless steel electiodes were placed transversely in the plantar area of the hindpaw contralateral to the perfused knee joint (approximately $10 \mathrm{~mm}$ apart). To determine stimulus intensities necessary to excite only large-diameter afferents, and intensities that also excite C-fibers, electrophysiological recordings were performed from bundles isolated from the sciatic nerve of pentobarbital anesthetized rats, as previously described (Ahlyren et al., 1992). Rats were anesthetized with pentobarbital sodium $(50 \mathrm{mg} / \mathrm{kg}$, i.p.), a tracheostomy performed and the trachea cannulated with PE-240 tubing. The rat was positioned ventral side down, and the skin over the posterior aspect of its thigh was incised and retracted to form a pool that was filled with warm $\left(37^{\circ} \mathrm{C}\right)$ mineral oil. The sciatic nerve was then exposed and dissected free of connective tissue in the mid-thigh. The nerve was crushed proximal to the recording site, the perineurium of the nerve was opened, and fine filaments dissected from the nerve, using sharpened jeweler's forceps. The filaments were placed on a silver-wire electrode for recording, and the reference electrode was attached to nearby tissue. Stimulating electrodes were placed transversely across the paw (see above) approximately $6 \mathrm{~cm}$ distal to the recording electrode. The stimulus intensities applied to the paw, which were found to reliably excite either A-fibers or A- and C-fibers, were 2.5 and $25 \mathrm{~mA}$. The inset in Figure 1 demonstrates that a $2.5 \mathrm{~mA}$ stimulus intensity is below the threshold for exciting $\mathrm{C}$-fibers, but above the threshold for exciting A-fibers, and that the $25 \mathrm{~mA}$ stimulus intensity also activate C-fibers.

Some rats received non-noxious (A-fiber strength) or noxious (C-fiber strength) electrical stimulation to the hindpaw during the assessment of BK-induced plasma extravasation: $25 \mathrm{~min}$ after the initiation of BK perfusion in the knee joint, rats received non-noxious $(2.5 \mathrm{~mA}, 0.25$ msec duration pulses, $3 \mathrm{~Hz}$ ) or noxious electrical stimulation $(25 \mathrm{~mA}$, $0.25 \mathrm{msec}$ duration pulses, $3 \mathrm{~Hz}$ ) via the two stimulating electrodes, which continued for the duration of the experiment, except in one group where the stimulation was stopped to determine the time course for recovery from inhibition. In one group of rats, transcutaneous electrical stimulation was applied to one forepaw.

\section{Surgical and pharmacological manipulations}

Acute surgical interruption of the lumbar sympathetic chain. The sympathetic chain was isolated bilaterally and loosely tied with two 2-0 silk threads, one at the level of $\mathrm{L}_{1-2}$ and the other at $\mathrm{L}_{2-3.3}$. The incision wounds were closed and sutured, and the animal prepared for knee joint perfusion. Acute sympathectomy was performed during knee joint perfusion by applying tension to the silk threads to interrupt the sympathetic chain. Perfusion of BK through the knee joint and electrical stimulation continued uninterrupted following acute surgical interruption of the lumbar sympathetic chain

Neonatal capsaicin. Destruction of small-diameter unmyelinated primary afferent fibers was achieved by administration of capsaicin (100 $\mathrm{mg} / \mathrm{kg}$ ) on postnatal day 1 . This method produces approximately an $85 \%$ depletion of unmyelinated primary afferent fibers (Nagy et al., 1983). Rats were subsequently used in plasma extravasation studies, when they weighed 300-350 gm (approximately 2 months postnatal).

Adrenalectomy. To remove both adrenal glands, rats were anesthetized with pentobarbital. An incision in the abdominal wall was made to expose the adrenal glands, which were then excised. Following surgery, rats received drinking water (ad libitum) containing $0.5 \%$ sodium chloride containing and $50 \mu \mathrm{g} / \mathrm{ml}$ corticosterone. Adrenalectomy was performed 1 week before plasma extravasation experiments.

Adrenal enucleation. To enucleate the adrenal gland (i.e., removing the adrenal medulla), under ether anesthesia, the adrenal gland was located through an incision in the lateral abdominal wall. Then the adrenal gland was exposed and the encapsulated adrenal medulla enucleated (Wilkinson et al., 1981). Rats then received drinking water (ad libitum) containing $0.5 \%$ sodium chloride for the first week after surgery. Adrenal enucleation was performed 5 weeks prior to the plasma extravasation experinents. A long postsurgical period was employed to allow HPA axis function to recover; however, in these rats, stress-induced changes in plasma corticosterone levels are still somewhat lower than in normal rats (Wilkinson et al., 1981).

Spinal transection. Spinal transection was performed on pentobarbital anesthetized rats. The spinal cord was exposed following incision along the dorsal midline and removal of the dorsal processes of the $T_{2}$ vertebra. The dura mater was opened and the spinal cord was transected using a blunt spatula. Surgery was performed one day hefore the plasma extravasation experiments.

Hypophysectomy. Hypophysectomized rats were purchased commercially from Charles River. Animals received $5 \%$ glucose in their drinking water for the first week after surgery, and were used in plasma extravasation experiments 2 weeks after surgery.

Metyrapone administration. Metyrapone (100 mg/kg), a corticosteroid synthesis inhibitor, was administered intraperitoneally (i.p.) $30 \mathrm{~min}$ before and $60 \mathrm{~min}$ after onset of BK perfusion.

\section{Materials}

Evans blue dye, bradykinin triacetate (BK), corticosterone (113,21-dihydroxy-4-pregnene-3,20-dione), platelet activating factor (PAF) ( $\mathrm{L}-\alpha-$ phosphatidylcholine, $\beta$-acetyl- $\tau$-o-alkyl), and dimethyl sulfoxide were obtained from Sigma Chemical Company, St. Louis, MO. Metyrapone was obtained from CIBA Geigy (Summit, NJ), and heat killed $M y c o-$ 


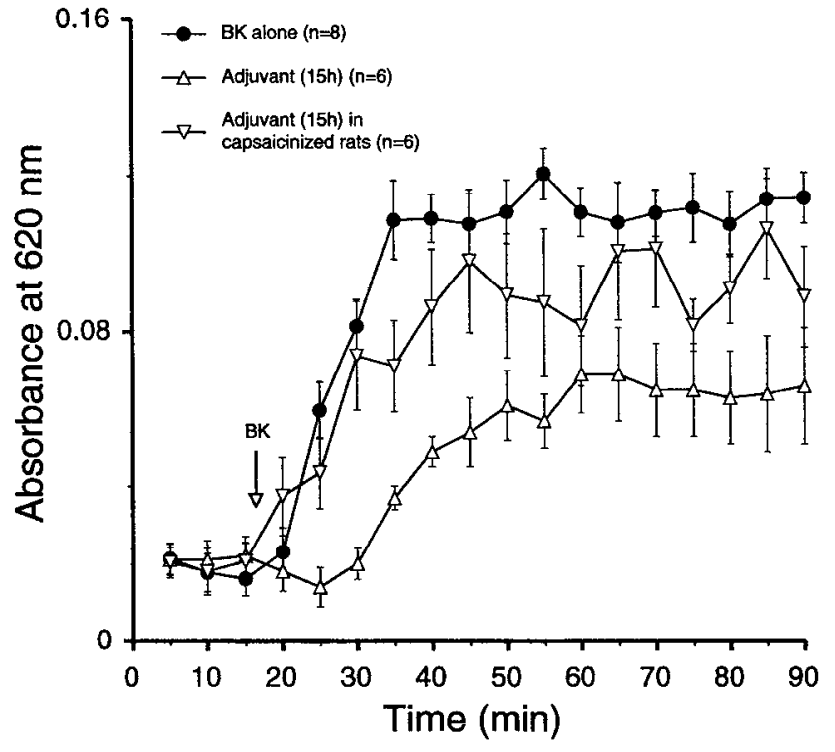

Figure 1. Effect of adjuvant pretreatment on BK-induced plasma extravasation in the rat knee joint. In all groups of rats, baseline plasma extravasation was established in the first three samples. In one group, BK ( $160 \mathrm{nM}$;,$n=8$ ) was then added to the perfusion fluid $(0.9 \%$ sodium chloride), and for the remainder of the experiment, was the only substance in the perfusion fluid. In a second group $(\triangle, n=6)$, rats had received $150 \mu \mathrm{l}$ of complete Freund's adjuvant injected subcutaneously in the paw $15 \mathrm{hr}$ before the start of the plasma extravasation experiment; as with the first group, BK was added after the first three baseline samples. Finally, a third group of rats that also received $150 \mu l$ of adjuvant in one hindpaw $15 \mathrm{hr}$ before the start of the plasma extravasation experiment, also were treated neonatally with capsaicin to destroy primary afferent C-fibers $(\nabla, n-6)$. In this and subsequent figures, data is presented as mean \pm SEM of $n$ values, and ordinate is absorbance of light at $620 \mathrm{~nm}$, which is linearly proportional to the concentration of Evans blue dye (Carr and Wilhelm, 1964). Once a drug is added to the perfusate, it continues to be present for the duration of the experiment.

bacterium butyricum (for Freund's adjuvant) was obtained from Difco, Detroit, MI. Corticosterone was dissolved in ethanol and diluted in saline to give a final concentration of 5\% ethanol. Metyrapone was dissolved in dimethyl sulfoxide. All other drugs were dissolved in $0.9 \%$ saline.

Data were analyzed using repeated measures analysis of variance. Fisher's least squares difference test (Гisher, 1949) was used for post hoc comparisons.

\section{Results}

\section{Peripheral afferent pathways}

Continuous perfusion of BK through the knee joint of the rat produces a sustained increase in plasma extravasation (Fig. 1, ; $n-8)$, which is first detected by $10 \mathrm{~min}$ after the onset of infusion of BK, reaches a plateau effect by $25 \mathrm{~min}$ and is sustained for at least $160 \mathrm{~min}$ (Green et al., 1993). To test if inflammation at a primary site (hindpaw) could inhibit inflammation at a secondary site (knee joint), complete Freund's adjuvant $(150 \mu \mathrm{l}, 10 \mathrm{mg} / \mathrm{ml})$ was injected into the hindpaw $15 \mathrm{hr}$ before BK perfusion of the knee joint. The experiments show that BK-induced plasma extravasation was markedly attenuated by prior injection of adjuvant in the paw (Fig. 1, $\triangle$ vs $P<$ 0.01). To test if this inhibition was elicited, at least in part, hy continuous activation of $\mathrm{C}$-fiber afferents from the inflamed site, adjuvant was injected $15 \mathrm{hr}$ prior to the onset of BK perfusion in rats that were treated neonatally with capsaicin. In these rats, $\mathrm{BK}$-induced plasma extravasation was not attenuated by the ad-

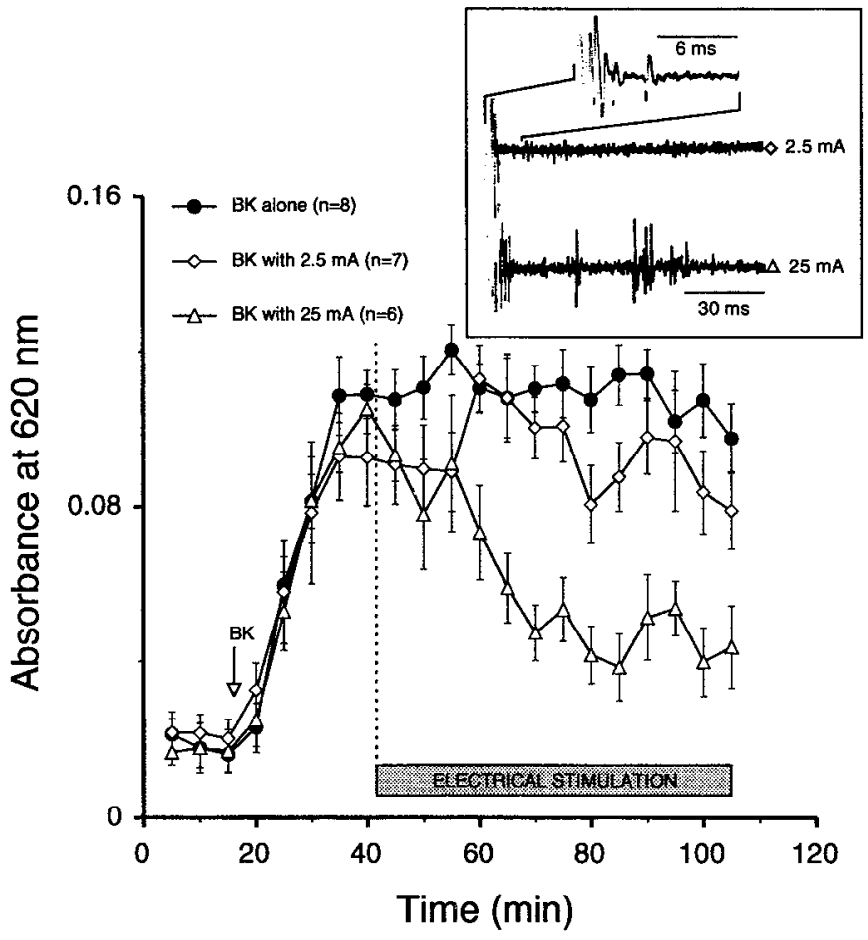

Figure 2. Effect of electrical stimulation on BK-induced plasma extravasation in the rat knee joint. Following establishment of baseline plasma extravasation in the first three samples, BK (160 nM;,$n=$ 8) was added to the perfusion fluid, and for the remainder of the experiment, was the only substance in the perfusion fluid. In a second group of rats ( $\triangle, n=6)$, C-fibers were stimulated electrically ( $25 \mathrm{~mA}$, $3 \mathrm{~Hz}$ ), via electrodes in one hindpaw, starting $40 \mathrm{~min}$ after the onset of the perfusion of $\mathrm{BK}$, while a third group $(\vartheta, n=6)$ had the same treatment as the second group, but received a lower intensity electrical stimulation, which did not activate C-fibers $(2.5 \mathrm{~mA}, 3 \mathrm{~Hz})$. Inset, Electrical activity recorded from fascicles dissected from the sciatic nerve of the rat during electrical stimulation of the hindpaw. The action potentials recorded in response to electrical stimulation $(0.25 \mathrm{msec}$ duration pulses) of the hindpaw of a rat is shown; the top trace was produced by a $2.5 \mathrm{~mA}$ stimulus pulse, while the bottom trace was produced by a $25 \mathrm{~mA}$ stimulus pulse. C-fiber action potentials are present only when the stimulation intensity was $25 \mathrm{~mA}$, while A-fiber action potentials are present in both traces. The top trace also includes an expanded timebase record to show A-fiber activation.

juvant-induced inflammation in the hindpaw (Fig. $1 ; \triangle$ vs $\nabla, P$ $<0.05 ; \nabla$ vs $\bigcirc, P>0.05)$.

We next tested whether activation of C-fibers from the site of primary inflammation could be responsible for the BK-induced plasma extravasation. We stimulated the paw electrically at stimulus intensities below and above C-fiber thresholds ( 2.5 and 25 $\mathrm{mA}$, respectively, both at $3 \mathrm{~Hz}$; Fig. 2 , inset). We found that $\mathrm{BK}$-induced plasma extravasation was inhibited by electrical stimulation (starting $25 \mathrm{~min}$ after addition of $\mathrm{BK}$ to the perfusing solution) when stimulation parameters were above C-fiber threshold, but not inhibited when the low intensity stimulus was employed (Fig. $2 ; \triangle$ vs $\bigcirc<P<0.05 ; \diamond$ vs $\bigcirc, P>0.05$ ). The depression of the BK-induced plasma extravasation was maximal approximately 25 min after the start of electrical stimulation; after termination of the electrical stimulation, BK-induced plasma extravasation recovered towards prestimulation levels after approximately $50 \mathrm{~min}$ (Fig. 3).

We also assessed the effect of activation of C-fibers on plasma extravasation induced by PAF; unlike BK, PAF-induced plasma 


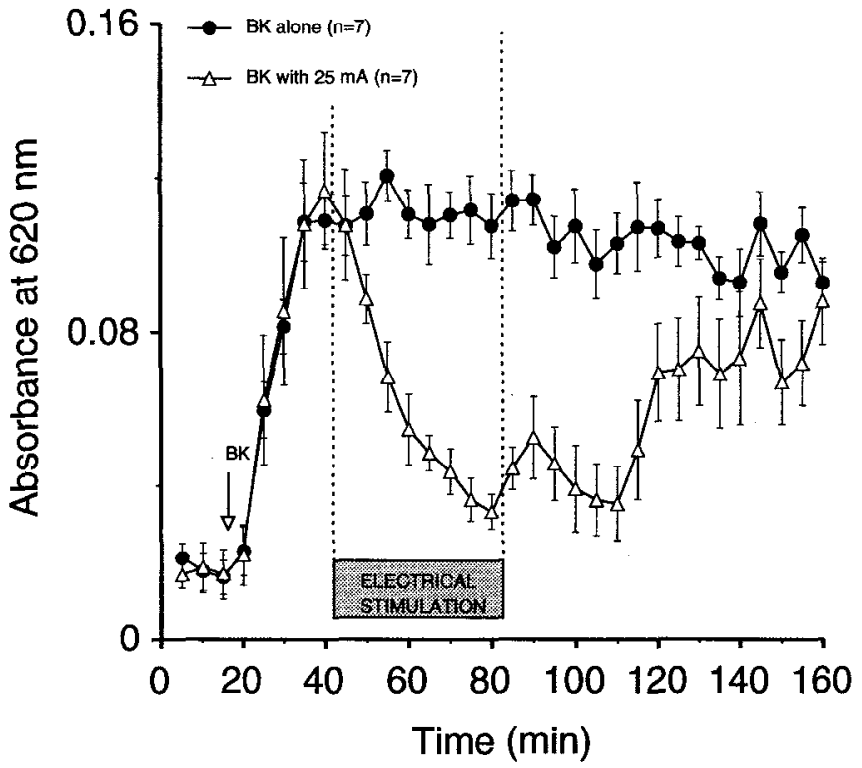

Figure 3. Time course for onset and recovery of electrical stimulationinduced inhibition of BK-induced plasma extravasation. Following establishment of baseline plasma extravasation in the first three samples, BK (160 nM;,$n=8$ ) was added to the perfusion fluid, and for the remainder of the experiment, was the only substance in the perfusion fluid. In a second group $(\triangle, n=6)$, C-fibers were stimulated electrically $(25 \mathrm{~mA}, 3 \mathrm{~Hz}$ ), via electrodes in one hindpaw, starting $40 \mathrm{~min}$ after the onset of the perfusion of $\mathrm{BK}$, and continuing for $40 \mathrm{~min}$; after this time, electrical stimulation was stopped while BK perfusion continued for an additional $80 \mathrm{~min}$.

extravasation is not dependent on the presence of sympathetic terminals (Green et al., 1993). The perfusion of PAF into the rat knee joint also increases plasma extravasation (Fig. 3). Unlike $\mathrm{BK}, \mathrm{PAF}$-induced plasma extravasation was not significantly inhibited by electrical stimulation (Fig. $4, \triangle$ vs $O ;<0.05$ ).

There is evidence that activation of small-diameter primary afferents causes activation of sympathetic efferents (Jänig et al., 1972; Sato and Schmidt, 1973) and that stimulation of sympathetic outflow inhibits plasma extravasation in the rat knee joint (Miao et al., unpublished observations). Therefore, we wanted to determine whether the observed inhibition of plasma extravasation by electrical stimulation of the hindpaw (Fig. 2) was due to activation of a reflex activation of the sympathetic supply of the knee joint. Therefore, we tested whether transcutaneous electrical activation of C-fibers also inhibited BK-induced plasma extravasation in the acutely sympathectomized rat, in which the postganglionic axons with their varicosities in the peripheral target are still intact. We found that inhibition of BK-induced plasma extravasation by electrical stimulation was not reversed after acute sympathectomy (Fig. $5, \diamond$ vs $\triangle ; P>0.05$ ), suggesting that reflex activation of sympathetic neurons is not responsible for this depression of plasma extravasation.

\section{Central pathways}

To test if ascending spinal pathways participate in the inhibition of BK-induced plasma extravasation induced by electrical stimulation, we transected the spinal cord at the level of $T_{2} 1 \mathrm{~d}$ prior to performing the perfusion experiment. Transection of the spinal cord prevented the inhibition of BK-induced plasma extravasation (Fig. $6, \nabla$ vs $O ; P>0.05$ ), suggesting that supraspinal mechanisms are involved in the inhibition of BK-induced plas-

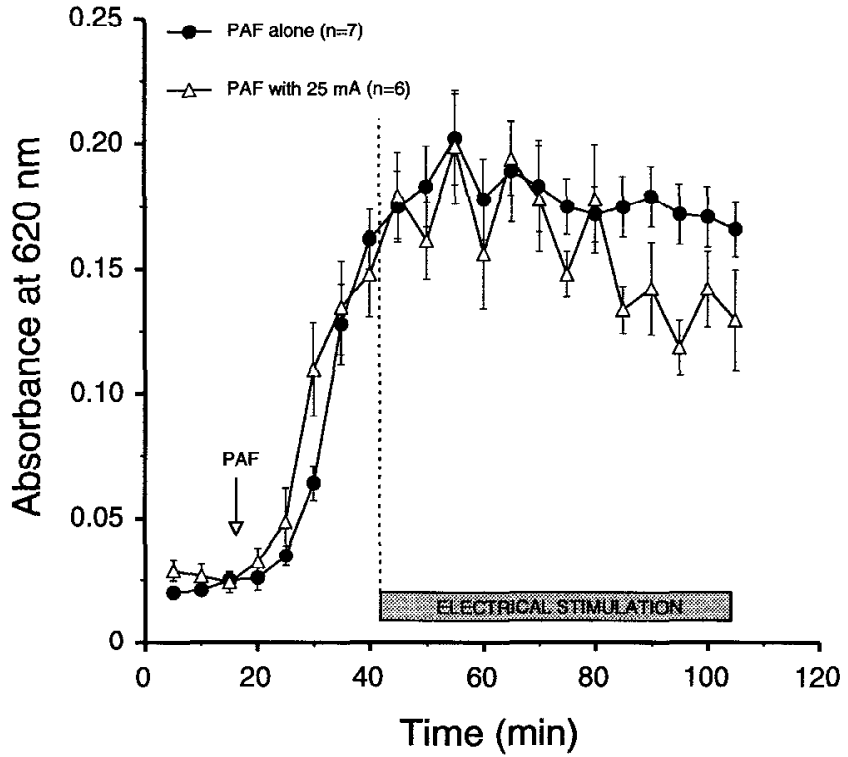

Figure 4. Effect of electrical stimulation on PAF-induced plasma extravasation in the rat knee joint. Following establishment of baseline plasma extravasation in the first three samples, PAF (100 nM;,$n=$ 7) was added to the perfusion fluid, and was the only substance in the perfusion fluid. In a second group $(\triangle, n=6)$, C-fibers were stimulated electrically $(25 \mathrm{~mA}, 3 \mathrm{~Hz})$ via electrodes in one hindpaw, starting 40 min after the start of the perfusion.

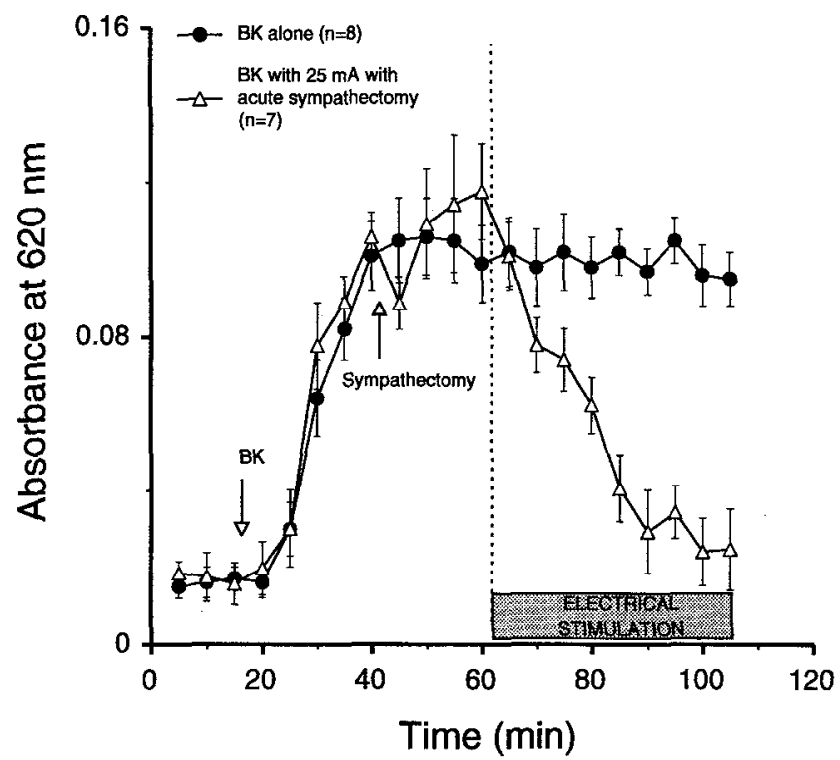

Figure 5. Effect of acute sympathectomy on electrical stimulationinduced inhibition of BK-induced plasma extravasation in the rat knee joint. Following establishment of baseline plasma extravasation in the first three samples, BK ( $160 \mathrm{nM}$; $n=8)$ was added to the perfusion fluid, and for the remainder of the experiment, was the only substance in the perfusion fluid in the control group of rats. In the second group of rats, BK (160 nM) was added after the third sample, and after the eighth sample, C-fibers were stimulated electrically $(25 \mathrm{~mA}, 3 \mathrm{~Hz})$, via electrodes in one hindpaw. The third group of rats $(\nabla, n=6)$ had undergone surgery to loosely attach 2-0 silk threads to the lumbar sympathetic chain (at $\mathrm{L}_{1-2}$ and $\mathrm{L}_{2-3}$ ); in this group, BK (160 nM) was added after the third sample, and after the eighth sample tension was applied to the threads to excise the sympathetic chain, thus producing an acute sympathectomy; after the twelfth sample, C-fibers were stimulated electrically $(25 \mathrm{~mA}, 3 \mathrm{~Hz})$, via electrodes in one hindpaw. 


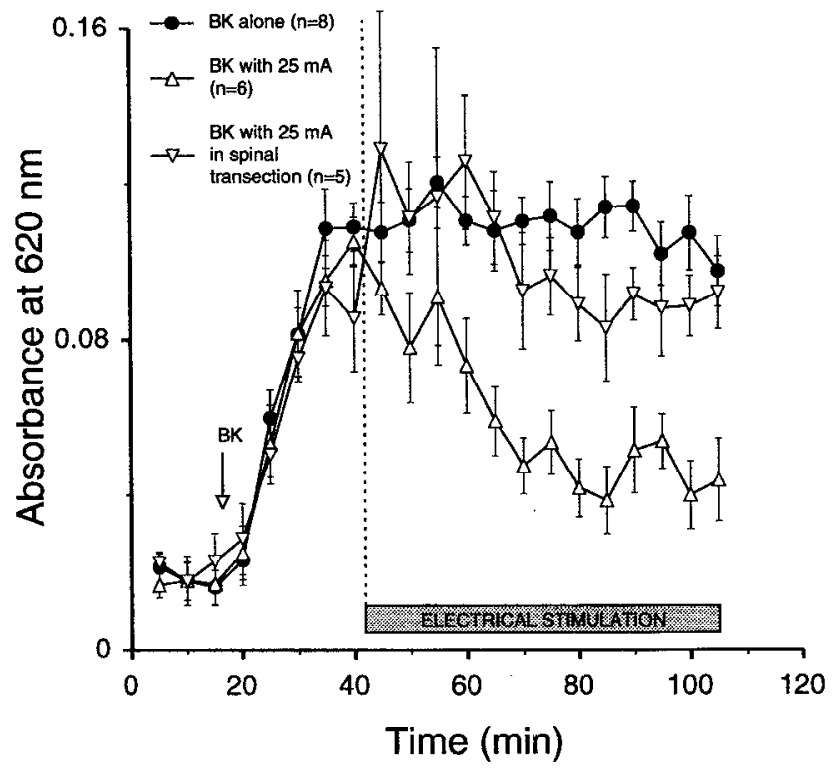

Figure 6. Effect of spinal transection on electrical stimulation-induced inhibition of BK-induced plasma extravasation in the rat knee joint. Following establishment of baseline plasma extravasation in the first three samples, BK (160 nм;,$n=8)$ was added to the perfusion fluid, and for the remainder of the experiment, was the only substance in the perfusion fluid in the control group of rats. In a second group of rats $(\triangle, n=6)$, BK $(160 \mathrm{nM})$ was added after the third sample, and after the eighth sample, C-fibers were stimulated electrically $(25 \mathrm{~mA}, 3 \mathrm{~Hz})$, via electrodes in one hindpaw. A third group of rats $(\nabla, n=5)$ had the same treatment as the second group, except that rats in this group had undergone surgery to transect the spinal cord at the thoracic level $\mathrm{T}_{2} 1 \mathrm{~d}$ before the start of the plasma extravasation experiment.

ma extravasation by activation of $\mathrm{C}$-fiber afferents, the major candidate being the HPA axis. We also studied the effect of electrical stimulation to the forepaw in normal and spinally transected rats. In both these groups electrical stimulation produced an attenuation of BK-induced plasma extravasation (Fig. 7; $\diamond$ vs $P<0.05$; $\nabla$ vs $P<0.05)$.

To evaluate the contribution of the HPA axis, we first studied the effect of selectively ablating specific components of this axis. In adrenalectomized rats, electrical stimulation did not inhibit BK-induced plasma extravasation (Fig. $8, \nabla$ vs $P>0.05$ ). Metyrapone, an inhibitor of glucocorticoid synthesis, also prevented the inhibition induced by electrical stimulation (Fig. 9, $\diamond$ vs $\quad ; P>0.05$ ). In hypophysectomized rats, noxious electrical stimulation did not inhibit BK-induced plasma extravasation (Fig. $10, \nabla$ vs $; P>0.05$ ). Since hypophysectomized rats were obtained from a supplier (Charles River) different to the other rats used in this study, we tested whether BK-induced plasma extravasation was the same in control Sprague--Dawley rats from Charles River and Bantin and Kingman. BK-induced plasma extravasation was the same in both Charles River and Bantin and Kingman Sprague-Dawley rats (data not shown).

In adrenal enucleated rats, inhibition of BK-induced plasma extravasation by noxious electrical stimulation was not statistically different from the inhibition seen in control rats ( $\triangle$ vs $\nabla$, $P>0.05$ ), although at some time points the inhibition is somewhat attenuated (Fig. 11). This might have been due to a slightly reduced function of the HPA axis in adrenal enucleated rats, as indicated by the slightly lower levels in plasma corticosterone at certain times of the day (Wilkinson et al., 1981). Based on

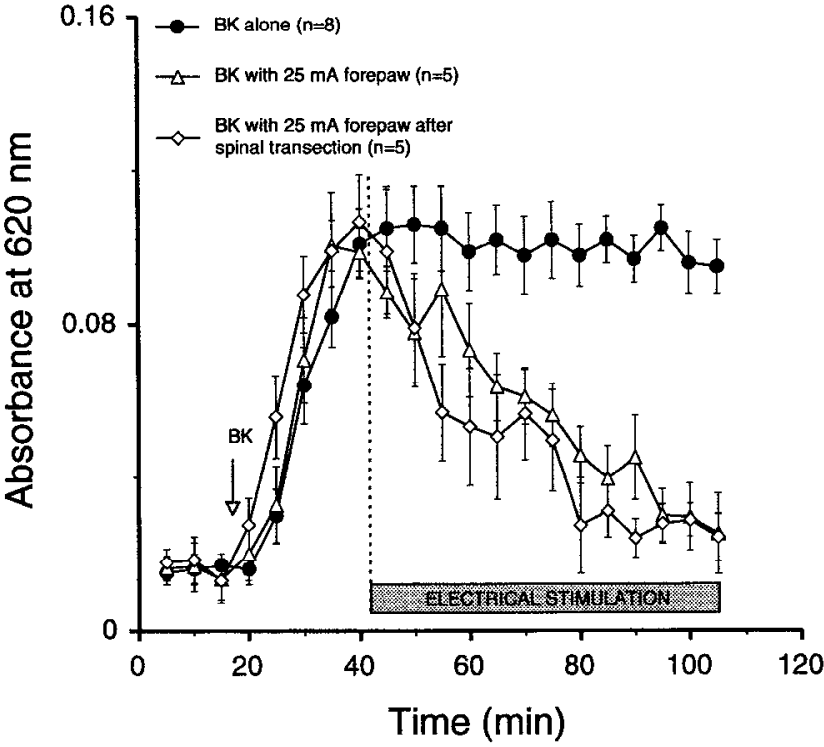

Figure 7. Effect of spinal transection on forepaw electrical stimulation-induced inhibition of BK-induced plasma extravasation in the rat knee joint. Following establishment of baseline plasma extravasation in the first three samples, BK (160 nM;,$n=8)$ was added to the perfusion fluid, and was the only substance in the perfusion fluid in the control group of rats. In a second group of rats $(\triangle, n=5)$, BK $(160$ nM) was added after the third sample, and after the eighth sample, C-fibers were stimulated electrically $(25 \mathrm{~mA}, 3 \mathrm{~Hz})$, via electrodes in one hindpaw. A third group of rats $(\diamond, n=5)$ had the same treatment as the second group, except that rats in this group had undergone surgery to transect the spinal cord at the thoracic level $T_{2} 1 \mathrm{~d}$ before the start of the plasma extravasation experiment.

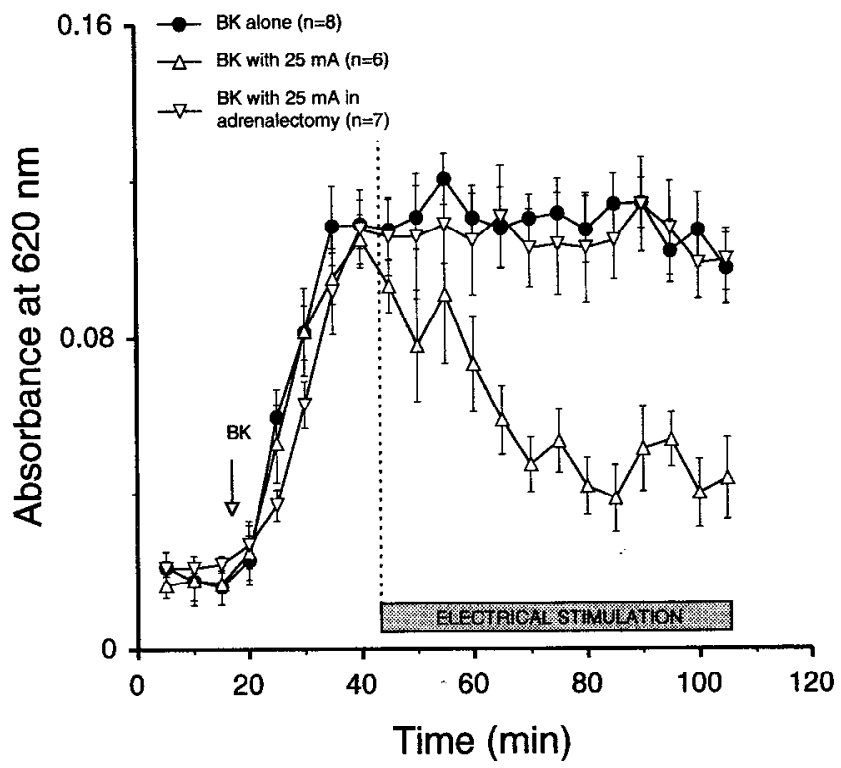

Figure 8. Effect of adrenalectomy on inhibition of BK-induced plasma extravasation in the rat knee joint produced by electrical stimulation of the hindpaw. Following establishment of baseline level of plasma extravasation, in the first three samples, BK $(160 \mathrm{nM} ; 0, n=8)$ was added to the perfusion fluid, and was the only substance in the perfusion fluid in the control group of rats. In a second group of rats, BK (160 nM) was added after the third sample, and after the eighth sample, C-fibers were stimulated electrically $(25 \mathrm{~mA}, 3 \mathrm{Iz})$, via clcctrodes in one hindpaw $(\Delta, n=7)$. A third group of rats $(\nabla, n=7)$ had the same treatment as the second group, but rats in this group had undergone surgical adrenalectomy 1 week before the plasma extravasation experiment. 


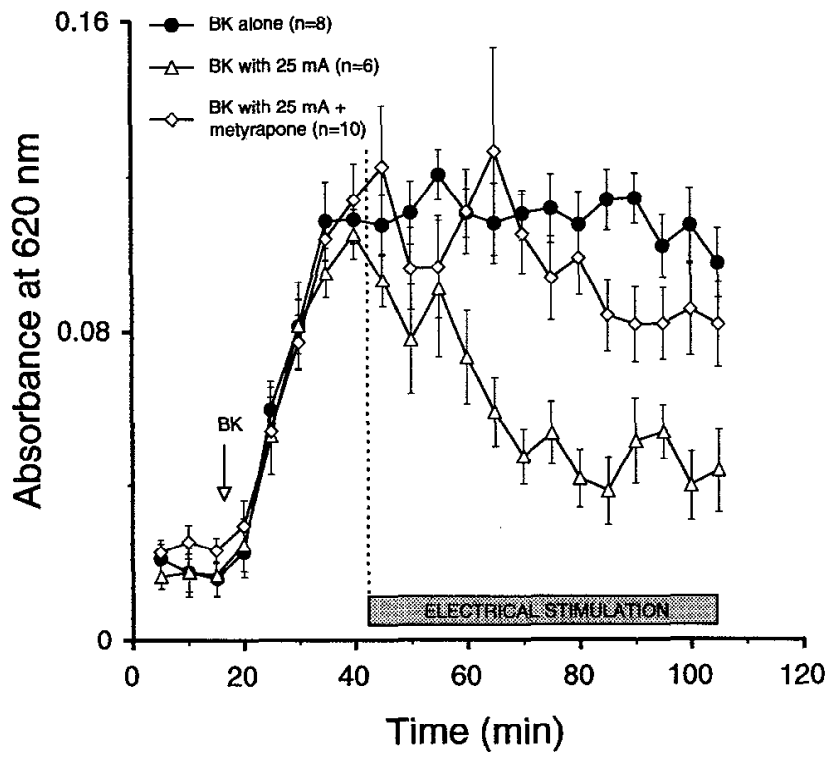

Figure 9. Effect of metyrapone (a corticosterone synthesis inhibitor) on inhibition of BK-induced plasma extravasation in the rat knee joint produced by electrical stimulation of the hindpaw. Following establishment of baseline plasma extravasation in the first three samples, BK (160 nM;,$n=8$ ) was added to the perfusion fluid, and for the remainder of the experiment was the only substance in the perfusion fluid. In a sccond group of rats $(\Delta, n=7)$, starting after the eighth sample, C-fibers were stimulated electrically $(25 \mathrm{~mA}, 3 \mathrm{~Hz})$, via electrodes in one hindpaw. In a third group of rats, which also received electrical stimulation after the eighth sample, metyrapone $(100 \mathrm{mg} / \mathrm{kg})$ was administered intraperitoneally twice, $30 \mathrm{~min}$ before and again 30 min after addition of $\mathrm{BK}(\diamond, n=10)$.

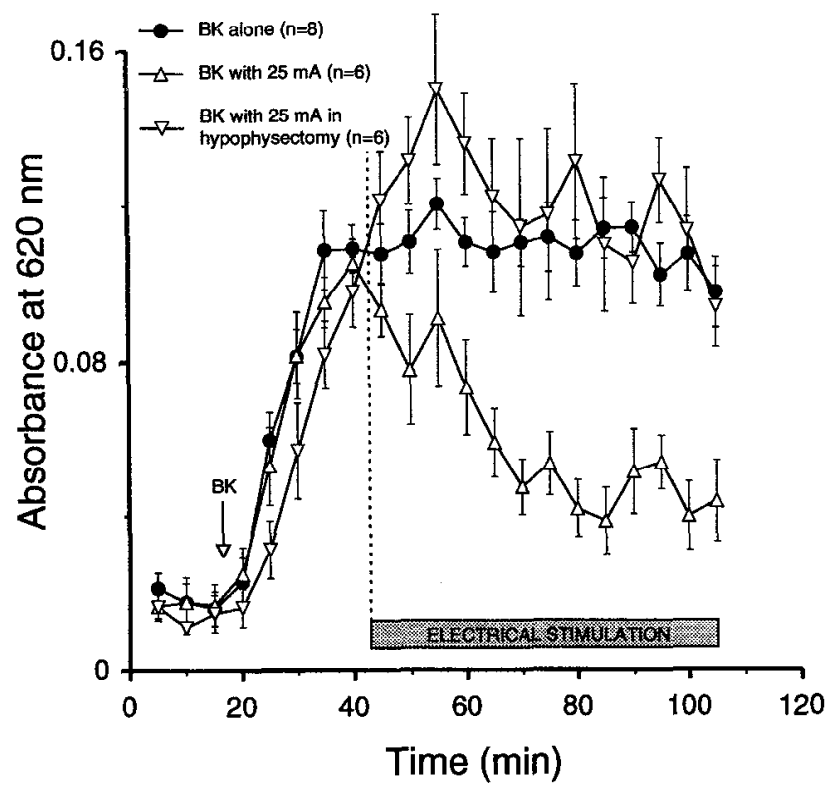

Figure 10. Effect of hypophysectomy on inhibition of BK-induced plasma extravasation in the rat knee joint produced by electrical stimulation of the hindpaw. Following establishment of baseline plasma extravasation in the first three samples, BK $(160 \mathrm{nM} ; \boldsymbol{O}, n=8)$ was added to the perfusion fluid, and for the remainder of the experiment, was the only substance in the perfusion fluid. In a second group of rats, BK (160 nM) was added after the third sample, and after the eighth sample, C-fibers were stimulated electrically $(25 \mathrm{~mA}, 3 \mathrm{~Hz})$, via electrodes in one hindpaw $(\triangle, n=6)$. A third group of rats $(\nabla, n=6)$ had the same treatment as the second group, but rats in this group had undergone surgical hypophysectomy.

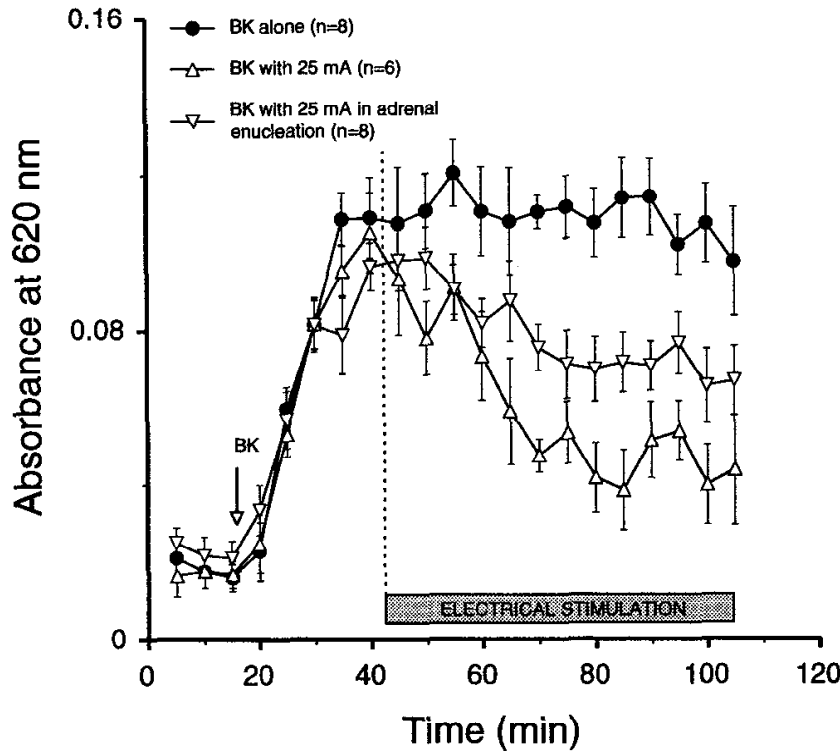

Figure 11. Fffect of adrenal enucleation on inhibition of BK-induced plasma extravasation in the rat knee joint produced by electrical stimulation of the hindpaw. Following establishment of baseline plasma extravasation in the first three samples, $\mathrm{BK}(160 \mathrm{nM} ; 0, n=8)$ was added to the perfusion fluid, and for the remainder of the experiment, was the only substance in the perfusion fluid. In the second group of rats, BK (160 nM) was added after the third sample, and after the eighth sample, C-fibers were stimulated electrically $(25 \mathrm{~mA}, 3 \mathrm{~Hz})$, via electrodes in one hindpaw. The third group of rats $(\nabla, n=8)$ had the same treatment as the second group, but rats in this group had undergone surgery to remove their adrenal medulla (adrenal enucleation), at least 3 weeks before the start of the plasma extravasation experiment.

the data described in Figures 1-11, we propose a schematic model of the circuit mediating feedback inhibition (Fig. 12).

\section{Discussion}

In this study we demonstrated that ongoing inflammation at one site in the body is able to inhibit a component of the inflammatory response (BK-induced plasma extravasation) at a second site. We also examined the mechanism of this ncgative control of the inflammatory response, and provide evidence that this phenomenon is mediated by a pathway involving $\mathrm{C}$-fiber afferents from the primary site of the inflammation, ascending tracts in the spinal cord and the HPA axis.

Freund's adjuvant-induced inflammation in the rat hindpaw attenuated BK-induced plasma extravasation in the knee joint; this attenuation was prevented in rats depleted of C-fibers by neonatal treatment with capsaicin. Similarly, acute electrical stimulation of the rat hindpaw at stimulus intensities which activate C-fibers (but not at lower stimulus intensities) also attenuated BK-induced plasma extravasation, and again this attenuation was prevented in C-fiber depleted rats (data not shown). These data implicate a role for $\mathrm{C}$-fiber primary afferents in feedback inhibition of inflammation. The inhibitory effect produced by $\mathrm{C}$-fiber activation appears to be mediated, in turn, by ascending pathways in the spinal cord, since we also showed that spinal transection prevented the attenuation of BK-induced plasma extravasation by electrical stimulation. In contrast, spinal transection $\left(\right.$ at $\mathrm{T}_{2}$ ) did not affect the inhibition of BK-induced plasma extravasation following transcutaneous stimulation of the rat forepaw (afferent input above $\mathrm{C}_{8}$ ), strongly suggesting an ascending pathway to supraspinal sites. We also show that feed- 


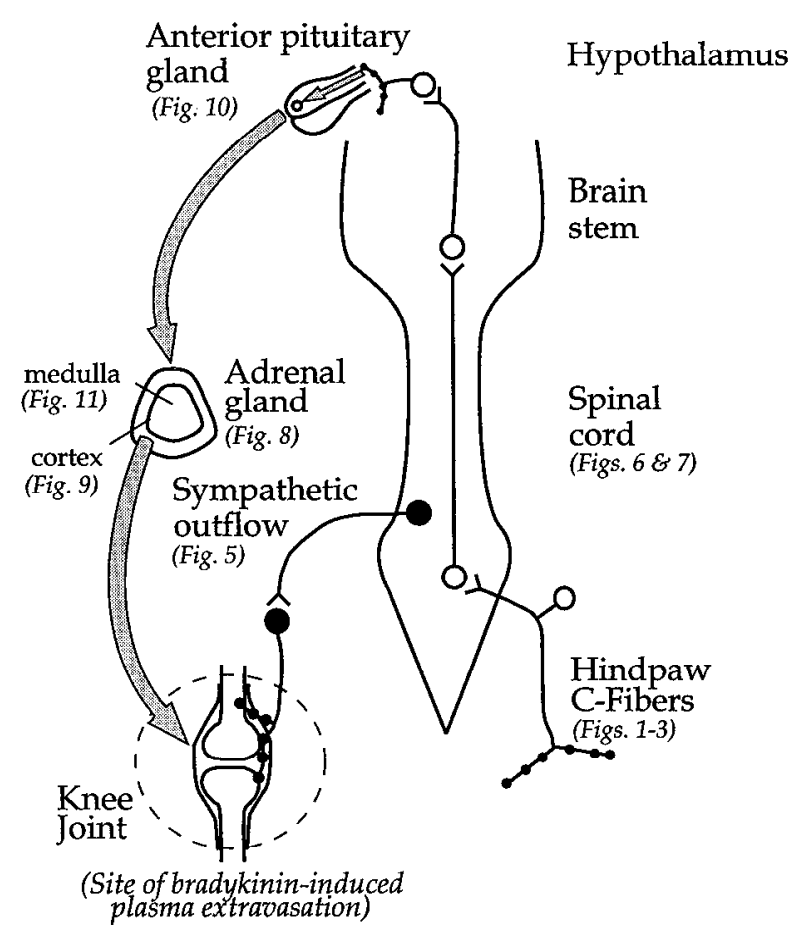

Figure 12. Schematic diagram illustrating hypothesized pathway by which an ongoing inflammatory response at one site produces a negative feedback inhibition on bradykinin-induced plasma extravasation at a second site. Activation of $\mathrm{C}$-fiber afferents occurs in inflamed tissue at the primary site (induced in our experiments by injecting Freund's adjuvant into the paw) and also during electrical stimulation of the hindpaw at intensities that excite C-fibers. Data shown in Figures 1-11 have provided evidence for likely components of the negative feedback pathway, and in this figure we have noted how each experiment and each preceding figure addresses the involvement of a particular component of the negative feedback circuit. The results of these experiments suggest that inhibition of plasma extravasation is mediated via a pathway which involves C-fiber activation of fibers in ascending tracts in the spinal cord which then stimulate the hypothalamus/pituitary, to release a mediator(s) (e.g., ACTH) which in turn stimulates the adrenal cortex to release glucocorticoids which act at the secondary site (knee joint).

back inhibition is not mediated via activation of sympathetic efferents, since acute sympathectomy, which does not affect the postganglionic axons and their varicose terminals in the joint capsule (at least for the duration of the experiment), did not affect the attenuation of BK-induced plasma extravasation by electrical stimulation. The time course to maximal suppression of BK-induced plasma extravasation (approximately $25 \mathrm{~min}$ ) and its recovery following termination (approximately $50 \mathrm{~min}$ ), suggests an involvement of humoral factors (rather than exclusively neuronal). Indeed, a subsequent step in this negative feedback pathway involves activation of the HPA axis, since the attenuation of BK-induced plasma extravasation by electrical stimulation is reduced or prevented in hypophysectomized and in adrenalcctomized rats, and in rats in which corticosterone synthesis was depressed. Two experiments argue against the adrenal medulla being involved in C-fiber activity-induced reduction of BK-induced plasma extravasation. Firstly, adrenal enucleation (removal of the adrenal medulla) did not significantly affect the inhibition of BK-induced plasma extravasation over the time measured. However, it should be pointed out that at some time points, electrical stimulation-induced inhibition of BK-induced plasma extravasation may be attenuated in the adrenal enucle- ated animals; this may be related to the observation that adrenal enucleation impairs adrenal cortical function, slightly decreasing resting evening and stress-induced plasma corticosterone levels (Wilkinson et al., 1981). Secondly, C-fiber stimulation of the forelimb in rats which were spinalized at the thoracic level $\mathrm{T}_{2}$ also led to a reduction of BK-induced plasma extravasation. The afferent input from the forelimb reaches the spinal cord rostral to the transection site and can therefore not influence the adrenal medulla via the sympathetic outflow distal to the transection site.

Our results suggest that the anti-inflammatory mediator of the HPA axis, corticosterone, may be a candidate mediator of the negative feedback inhibitory system that inhibits BK-induced plasma extravasation in the joint, since administration of the glucocorticoid synthesis inhibitor, metyrapone, prevented the attenuation of BK-induced plasma extravasation by electrical stimulation. However, our data do not exclude the possibility that other mediators of the HPA axis are also involved.

The data provided in this study suggests a circuit whereby inflammatory processes activate C-fiber afferents which, via spinal cord pathways, activates the HPA axis, leading to the subsequent release of glucocorticoids. The evidence that a neuronal component participates in the control of inflammation differs from previous models which have primarily focused on the role of mediators (such as CRF) released at the site of inflammation and/or in the CNS (Garcia-Leme and Schapoval, 1975; Moraes and Garcia-Leme, 1982; Wei and Kiang, 1989; Wei et al., 1990; Gao et al., 1991; Wei and Gao, 1991). For example, if a carrageenan inflamed paw is perfused with saline, the perfusate has potent anti-inflammatory activity when injected intravenously into a donor rat; it has been shown that this effect is also dependent on the HPA axis, since hypophysectomy or adrenalectomy prevents the formation of the anti-inflammatory substance(s) (Garcia-Leme and Schapoval, 1975). Furthermore, while CRF's ability to attenuate the inflammatory response has been attributed to actions of humoral mediators (Smith et al., 1986; Zelazowski et al., 1992), there are other reports which suggest that there is a neuronal component of feedback inhibition. Thus, plasma corticostcrone levels are increased in response to nociceptive input (i.e., activation of C-fibers) (Stenberg et al., 1990). Additional evidence for a neuroendocrine negative feedback inhibition of the inflammatory process, mediated via primary C-fiber afferents, comes from one study, in which injection of formalin (which both activates C-fibers and produces inflammation) in the rat paw activates a supraspinal neuronally mediated feedback inhibitory system that is dependent on descending neuronal pathways and/or a humoral component (Wheeler-Aceto and Cowan, 1991), and another study, in which intraperitoneally administered formalin produces an increase in plasma ACTH in normal, but not in neonatally capsaicin treated, rats (Amann and Lembeck, 1987).

The data presented here demonstrates that unlike BK, PAFinduced plasma extravasation is not attenuated by electrical stimulation. Since we have previously shown that BK-induced plasma extravasation is dependent on the sympathetic postganglionic neuron (Coderre et al., 1989) while PAF-induced plasma extravasation is not neuronal-dependent (Green et al., 1993) and likely to induce plasma extravasation by direct action on the postcapillary venules (Braquet et al., 1987), the observation that BKand PAF-induced plasma extravasation are differentially affected by electrical stimulation implicates the sympathetic postganglionic neuron terminal as a target for feedback inhibition. The data we have presented implicate the HPA axis in negative feed- 
back inhibition, and in particular the role of corticosterone, with the sympathetic postganglionic neuron terminal as its target, to inhibit BK-induced plasma extravasation. A possible mechanism of action may be via inhibition of prostaglandin synthesis, since we have shown that BK-induced plasma extravasation is dependent on the production of prostaglandins (Coderre et al., 1989), and glucocorticoids attenuate prostaglandin synthesis by inhibiting the activity of both phospholipase A2 and cyclo-oxygenase isomerase (Goppelt-Struebe et al., 1989). Additional studies are required to determine whether HPA mediators of feedback inhibition act on the sympathetic post-ganglionic neuron terminal, and what mediators are the ultimate components of the feedback inhibition circuit. We are currently investigating the effect of infusion of corticosterone directly into the knee joint on the magnitude of BK-induced plasma extravasation.

In conclusion, we have demonstrated that inflammation attenuates the ability to produce a component of inflammation (plasma extravasation) at a second site. This observation appears to reflect a normal physiological response whereby ongoing inflammation is modulated (negative feedback). Furthermore, we have demonstrated that this negative feedback system includes a neuronal and endocrine component, specifically C-fiber primary afferents, spinal pathways, and the HPA axis.

\section{References}

Ahlgren SC, White DM, Levine JD (1992) Increased responsiveness of sensory neurons in the saphenous nerve of the streptozotocindiabetic rat. J Neurophysiol 68:2077-2085.

Amann R, Lembeck F (1987) Stress induced ACTH release in capsaicin treated rats. Br J Pharmacol 90:727-731.

Barnes P (1991) Neurogenic inflammation in airways. Appl Immunol 94:303-309.

Basbaum AI, Levine JD (1991) The contribution of the nervous system to inflammation and inflammatory disease. Can J Physiol Pharmacol 69:647-651.

Braquet P, Touqui L, Shen TY, Vargaftig BB (1987) Perspectives in platelet-activating factor research. Pharmacol Rev 39:97-145.

Brezinschek HP, Faessler R, Klocker H, Kroemer G, Sgonc R, Dietrich H, Jakober R, Wick G (1990) Analysis of the immune-endocrine feedback loop in the avian system and its alteration in chickens with spontaneous autoimmune thyroiditis. Eur J Immunol 20:2155-2159.

Calogero AE, Sternberg EM, Bagdy G, Smith C, Bernardini R, Aksentijevich S, Wilder RT, Gold PW, Chrousos GP (1992) Neurotransmitter-induced hypothalamic-pituitary-adrenal axis responsiveness is defective in inflammatory disease-susceptible Lewis rats: in vivo and in vitro studies suggesting globally defective hypothalamic secretion of corticotropin-releasing hormone. Neuroendocrinology 55:600-608.

Carr J, Wilhelm DL (1964) The evaluation of increased vascular permeability in the skin of guinea pigs. Aust J Exp Biol Med Sci 42: 511-522.

Casley-Smith JR, Window J (1976) Quantitative morphological correlations of alterations in capillary permeability, following histamine and moderate burning, in the mouse diaphragm; and the effects of benzopyrones. Microvasc Res 11:279-305.

Chikanza IC, Petrou P, Kingsley G, Chrousos G, Panayi GS (1992) Defective hypothalamic response to immune and inflammatory stimuli in patients with rheumatoid arthritis. Arthritis Rheum 35:12811288.

Coderre TJ, Basbaum AI, Levine JD (1989) Neural control of vascular permeability: interactions between primary afferents, mast cells, and sympathetic efferents. J Neurophysiol 62:48-58.

Dunn AJ (1993) Infection as a stressor: a cytokine-mediated activation of the hypothalamo-pituitary-adrenal axis? CIBA Found Symp 172: 226-239.

Fisher RA (1949) The design of experiments. Edinburgh: Oliver and Boyd.

Gao GC, Dashwood MR, Wei ET (1991) Corticotropin-releasing factor inhibition of substance P-induced vascular leakage in rats: possible sites of action. Peptides 12:639-644.

Garcia-Leme J, Schapoval EE (1975) Stimulation of the hypothalamo- pituitary-adrenal axis by compounds formed in inflamed tissue. $\mathrm{Br} . \mathrm{J}$ Pharmacol 53:75-83.

Garcia-Leme J, Fortes ZB, Sannomiya P, Farsky SP (1992) Insulin, glucocorticoids and the control of inflammatory responses. Agents Actions [Suppl] 36:99-118

Goppelt-Struebe M, Wolter D, Resch K (1989) Glucocorticoids inhibit prostaglandin synthesis not only at the level of phospholipase A2 but also at the level of cyclo-oxygenase/PGE isomerase. $\mathrm{Br} \mathbf{J}$ Pharmacol 98:1287-1295

Green PG, Luo J, Heller PH, Levine JD (1993a) Neurogenic and nonneurogenic mechanisms of plasma extravasation in the rat. Neuroscience 52:735-743.

Green PG, Luo J, Heller PH, Levine JD (1993b) Further substantiation of a significant role for the sympathetic nervous system in inflammation. Neuroscience 55:1037-1043.

Harbuz MS, Rees RG, Lightman SL (1993) HPA axis responses to acute stress and adrenalectomy during adjuvant-induced arthritis in the rat. Am J Physiol 264:R179-85.

Haskill S, Yurochko AD, Isaacs KL (1992) Regulation of macrophage infiltration and activation in sites of chronic inflammation. Ann NY Acad Sci 664:93-102.

Jänig W, Sato A, Schmidt RF (1972) Reflexes in postganglionic cutaneous fibres by stimulation of group I to group IV somatic afferents. Pfluegers Arch 331:244-256.

Keshav S, Chung LP, Gordon S (1990) Macrophage products in inflammation. Diagn Microbiol Infect Dis 13:439-447.

Khalil Z, Helme RD (1989) Sympathetic neurons modulate plasma extravasation in the rat through a non-adrenergic mechanism. Clin Exp Neurol 26:45 50 .

Kidd BL, Cruwys S, Mapp PI, Blake DR (1992) Role of the sympathetic nervous system in chronic joint pain and inflammation. Ann Rheum Dis 51:1188-1191.

Kowalski ML, Kaliner MA (1988) Neurogenic inflammation, vascular permeability, and mast cells. J Immunol 140:3905-3911.

Levine JD, Goetzl EJ, Basbaum AI (1987) Contribution of the nervous system to the pathophysiology of rheumatoid arthritis and other polyarthritides. Rheum Dis Clin N Am 13:369-383.

Mantyh PW, Catton MD, Boehmer CG, Welton ML, Passaro EP Jr, Maggio JE, Vigna SR (1989) Receptors for sensory neuropeptides in human inflammatory diseases: implications for the effector role of sensory neurons. Peptides 10:627-645.

Moraes FR, Garcia-Leme J (1982) Endogenous corticosteroids and insulin in acute inflammation. Microvasc Res 23:281-293.

Nagy JI, Iversen LL, Goedert M, Chapman D, Hunt SP (1983) Dosedependent effects of capsaicin on primary sensory neurons in the neonatal rat. J Neuropharmacol 3:399-406.

Neeck G, Federlin K, Graef V, Rusch D, Schmidt KL (1990) Adrenal secretion of cortisol in patients with rheumatoid arthritis. J Rheumatol 17:24-29.

Neidhart M, Fluckiger EW (1992) Hyperprolactinaemia in hypophysectomized or intact male rats and the development of adjuvant arthritis. Immunology 77:449-455.

Payne LC, Krueger JM (1992) Interactions of cytokines with the hypothalamus-pituitary axis. J Immunother 12:171-173.

Roos D, Dolman KM (1990) Neutrophil involvement in inflammatory tissue damage. Neth J Med 36:89-94.

Sato A, Schmidt RF (1973) Somatosympathetic reflexes: afferent fibers, central pathways, discharge characteristics. Physiol Rev 53: 916-947.

Smith EM, Morrill AC, Meyer WJ III, Blalock JE (1986) Corticotropin releasing factor induction of leukocyte-derived immunoreactive ACTH and endorphins. Nature 321:881-882.

Stenberg VI, Bouley MG, Katz BM, Lee KJ, Parmar SS (1990) Negative endocrine control system for inflammation in rats. Agents Actions 29:189-195.

Sternberg EM, Hill JM, Chrousos GP, Kamilaris T, Listwak SJ, Gold PW, Wilder RL (1989a) Inflammatory mediator-induced hypothalamic-pituitary-adrenal axis activation is defective in sireplococcal cell wall arthritis-susceptible Lewis rats. Proc Natl Acad Sci USA 86:2374-2378.

Sternberg EM, Young WB, Bernardini R, Calogero AE, Chrousos GP, Gold PW, Wilder RL (1989b) A central nervous system defect in biosynthesis of corticotropin-releasing hormone is associated with susceptibility to streptococcal cell wall-induced arthritis in Lewis rats. Proc Natl Acad Sci USA 86:4771-4775. 
Sternberg EM, Wilder RL, Gold PW, Chrousos GP (1990) A defect in the central component of the immune system-hypothalamic-pituitaryadrenal axis feedback loop is associated with susceptibility to experimental arthritis and other inflammatory diseases. Ann NY Acad Sci 594:289-292.

Sternberg EM, Chrousos GP, Wilder RL, Gold PW (1992) The stress response and the regulation of inflammatory disease. Ann Intern Med 117:854-866.

Sweep F, Rijnkels C, IIermus A (1991) Activation of the hypothalamus-pituitary-adrenal axis by cytokines. Acta Endocrinol 125[Suppl 1]:84-91.

Wei ET, Gao GC (1991) Corticotropin-releasing factor: an inhibitor of vascular leakage in rat skeletal muscle and brain cortex after injury. Regul Pept 33:93-104.

Wei ET, Kiang JG (1989) Peptides of the corticoliberin superfamily attenuate thermal and neurogenic inflammation in rat pawskin. Eur J Pharmacol 168:81-86.
Wei ET, Wong JC, Kiang JG (1990) Decreased inflammatory responsiveness of hypophysectomized rats to heat is reversed by a corticotropin-releasing factor (CRF) antagonist. Regul Pept 27:317-323.

Wheeler-Aceto H, Cowan A (1991) Neurogenic and tissue-mediated components of formalin-induced edema: evidence for supraspinal regulation. Agents Actions 34:264-269

Wilkinson CW, Shinsako J, Dallman MF (1981) Return of pituitaryadrenal function after adrenal enucleation or transplantation: diurnal rhythms and responses to cther. Endocrinology 109:162-169.

Williams TJ, Brain SD, Hellewell PG, Jose PJ, Nourshargh S, Rampart M (1988) Alteration in microvascular permeability induced by products released during inflammation. Prog Clin Biol Res 263:55-69.

Zelazowski P, Smith MA, Gold PW, Chrousos GP, Wilder RL, Sternberg EM (1992) In vitro regulation of pituitary ACTH secretion in inflammatory disease susceptible Lewis (LEW/N) and inflammatory disease resistant Fischer (F344/N) rats. Neuroendocrinology 56:474482 . 